\title{
Reconstruction of Initial Wave Field of a Nonsteady-State Wave Propagation from Limited Measurements at a Specific Spatial Point Based on Stochastic Inversion
}

\author{
S. L. Han and Takeshi Kinoshita \\ Institute of Industrial Science, The University of Tokyo, 4-6-1 Komaba, Meguro-ku, Tokyo 153-8505, Japan \\ Correspondence should be addressed to Takeshi Kinoshita; kinoshit@iis.u-tokyo.ac.jp
}

Received 1 August 2012; Revised 11 March 2013; Accepted 14 March 2013

Academic Editor: Fatih Yaman

Copyright (C) 2013 S. L. Han and T. Kinoshita. This is an open access article distributed under the Creative Commons Attribution License, which permits unrestricted use, distribution, and reproduction in any medium, provided the original work is properly cited.

\begin{abstract}
This paper studies an inverse problem that can be used for reconstructing initial wave field of a nonsteady-state wave propagation. The inverse problem is ill posed in the sense that small changes in the input data can greatly affect the solution of the problem. To address the difficulty, the problem is formulated in the form of an inference problem in an appropriately constructed stochastic model. It is shown that the stochastic inverse model enables the initial surface disturbance to be reconstructed, including its confidence intervals given the noisy measurements. The reconstruction procedure is illustrated through applications to some simulated data for two- and three-dimensional problem.
\end{abstract}

\section{Introduction}

Water waves are one of important subjects in ocean-related fields. They are generated on the free surface, between air and water, from various sources such as a storm at sea, moving ship in calm water, and impact from a falling object. Once they are generated, wave propagation occurs on the surface of water away from the source. The propagation of water wave is a little more complex than many other kinds of waves in nature due to its dispersion.

In this paper, we are interested in a nonsteady-state water wave propagation generated by an initial surface disturbance. The classical problem in water waves is the computation of the time and spatial evolution in a whole body of water with this given initial wave field. That is, the aim of such problems is to predict the propagation of water waves as effects of the initial free surface disturbance, which is the cause of this phenomenon. This kind of problem has been widely studied because of its importance [1-6]. We name it forward or direct problem. Although much progress has been achieved in the analysis of resulting wave flow, there are only few studies [711] on inverse problems, whose aim is to find the cause of the wave propagation.
In this study, our attention is focused on the inverse problem of reconstructing initial wave field from the limited measurement on the specific boundary of the fluid body for two- and three-dimensional problems. Based on the assumption of linear dispersive wave theory, the reconstructing problem can be formulated as an inverse problem. The problem requires solving the Fredholm integral equation of the first kind, which causes severe ill posedness when numerically approximating. As a result, the inverse problem (reconstruction of initial wave field) is mathematically and numerically much more challenging than the forward problem (simulation of future wave field given initial disturbance). For such problems, the solution may not exist when noise is present in the measurements. Moreover, it may also loses stability although it exists, that is, lacks continuous dependence on the data. Thus, a small perturbation of the data may yield erroneous results of the inverse solution.

To overcome the difficulties, we adopt a stochastic inverse method, which has recently attracted much research attention in diverse fields [12-15]. Stochastic inverse methods solve the inverse problem in a systematic way by modeling all parameters of interests as random variables with joint probability distributions. This randomness can be considered as 
parameter variability since it is related to the uncertainty of the true values. The key idea behind stochastic inverse method is to restate the original inverse problem in the form of probabilistic description of the unknown parameters, that is, stochastic inverse problem. Accordingly, the solution of the inverse problem is represented by a probability distribution of random unknowns. This probability distribution is known as the posterior probability distribution function incorporating the degree of confidence of unknown parameter given the measurement.

Two aspects of this study are worth noting. Firstly, the reconstruction procedure was derived from the limited measurement on the specific boundary of the fluid body. Secondly, a stochastic inverse method is introduced to enable the stable reconstruction procedure, since it requires solving the ill-posed problem. It also provides a way of quantifying an uncertainty in the solution, as well as smoothing the solution by expanding original solution spaces into probability spaces.

This study is organized as follows. Section 2 reviews twoand three-dimensional problems regarding a nonsteady-state water wave propagation due to initial surface disturbance. In Section 3, a procedure is described for the reconstruction of the initial wave field based on the limited measurements at a fixed spatial point. The stochastic inversion method is illustrated in Section 4 for the stable solution procedure. In Section 5, the reconstruction procedure is validated through applications to some digitally simulated data. Finally, we make concluding remarks in Section 6.

\section{Water Waves due to an Initial Impulse}

2.1. Mathematical Description of the Problem. It is assumed here that the motion of water waves is described by the Euler equations [1-6]. That is, the fluid is homogeneous, incompressible, and inviscid. The water depth is constant $z=$ $-h$, and the surface tension is neglected. In this context, the corresponding fluid motion $\varphi(x, y, z, t)$ is governed by the Laplace equation

$$
\nabla^{2} \varphi=0 \text { in the water }
$$

with the linearized boundary conditions

$$
\begin{gathered}
\frac{\partial \eta}{\partial t}=\frac{\partial \varphi}{\partial z} \text { on the surface, } \\
\frac{\partial \varphi}{\partial t}+g \eta=0 \text { on the surface, } \\
\frac{\partial \varphi}{\partial z}=0 \text { on the bottom, }
\end{gathered}
$$

where $\eta, g$ denote the surface elevation, gravitational acceleration, respectively.

The Euler equations can mathematically be viewed as a Cauchy problem [6], studying a partial differential equation that satisfies certain conditions which are given on a hypersurface in the domain. Given the initial spatial surface disturbance at time zero $\eta(x, y, 0)$ or $\varphi(x, y, z, 0)$, the above equations give a way to determine the whole behavior of fluid with respect to time and space.

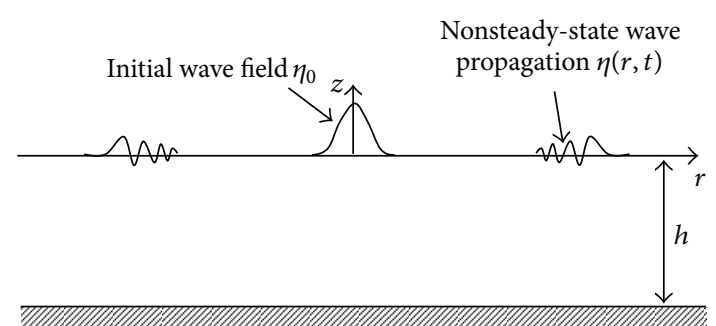

Figure 1: Wave propagation generated by the initial disturbance $\eta_{0}$ on the water surface.

2.2. Surface Waves due to Localized Impulse. If the initial wave disturbance $\eta_{0}$, as shown in Figure 1 , is generated by the localized sudden disturbances such as underwater explosions or sudden vertical seafloor displacement, the resulting nonsteady-state waves can be described by the axisymmetric form $[1-6,8,9]$

$$
\eta(r, t)=\int_{0}^{\infty} a(k) k J_{0}(k r) \cos \omega t d k,
$$

where $r=\sqrt{x^{2}+y^{2}}, J_{0}(\cdot)$ is the usual Bessel function of the first kind of order zero, $k$ is the wave number, and $\omega$ is wave frequency. Two parameters $k, \omega$ are related to each other through the dispersion relation $\omega^{2}=k g \tanh (k h)$. The coefficient function $a(k)$ is determined with the initial wave disturbance $\eta_{0}$, which is defined by spatial wave field when $t=0$, by the Hankel transform:

$$
a(k)=\int_{0}^{\infty} \eta_{0}(r) r J_{0}(k r) d r
$$

If the Hankel transform of the initial wave disturbance $\eta_{0}$ is known, the resulting wave motion can be predictable with (3).

For a two-dimensional system $(x, z, t)$, the problem for the nonsteady-state waves can be simply described [1-6] by

$$
\eta(x, t)=\int_{-\infty}^{\infty} a(k) \exp \{i(k x \pm \omega t)\} d k,
$$

where the coefficient function defined by the Fourier transform of $\eta(x, 0) / 2$ is

$$
a(k)=\frac{1}{2 \pi} \int_{-\infty}^{\infty} \frac{\eta_{0}}{2} e^{-i k x} d x .
$$

2.3. Forward and Inverse Problems. If the initial wave disturbance $\eta_{0}$ is specified, the coefficient function $a(k)$ can be directly obtained by the Hankel transform (4) for the three-dimensional problem and the Fourier transform (6) for the two-dimensional problem. The wave evolution $\eta$ with respect to space and time variables is then simply computed by integrating (3) or (5) for the given coefficient function. The computation of the wave evolution from knowledge of the initial wave disturbance is defined as the forward problem.

On the other hand, the inverse problem of reconstructing initial wave field on the basis of the measured wave elevation on the water surface is not simple. Such inverse procedures 
often involve ill-posed problems whose solution does not exist or lacks continuous dependence on the given data [711]. Thus, the problems are mathematically and numerically much more challenging than the forward problem. In the present study, the aim is focused to reconstruct the initial wave field given a wave history data recorded at a specific spatial point.

\section{Reconstruction of Initial Wave Field}

3.1. Reconstructing Procedure. The inverse procedure for reconstructing initial wave field can be achieved by two steps: (i) estimating the coefficient function $a(k)$ based on the measurement wave elevation at a fixed point, and (ii) transforming the estimated coefficient function into the initial wave field.

Formally, the first step for the reconstruction can be expressed as an operator equation:

$$
H\{a(k)\}=\eta_{M}(t),
$$

where $a(k)$ denotes the unknown coefficient function with a finite interval $|k|<N$ and $\eta_{M}(t)$ denotes the wave elevation measured at fixed-spatial point during the time interval $0<$ $t<T$ and the operator $H: a \rightarrow \eta_{M}$ is defined by

$$
H\{\cdot\}= \begin{cases}\int_{-N}^{N} e^{i(k X \pm \omega t)}\{\cdot\} d k, & \text { for 2D problem, } \\ \int_{0}^{N} k J_{0}(k R) \cos (\omega t)\{\cdot\} d k, & \text { for 3D problem. }\end{cases}
$$

In (8), $X$ and $R$ represent specific measurement points for $2 \mathrm{D}$ problem and $3 \mathrm{D}$ problem, respectively.

Let us denote a surface elevation measured at time $t_{i}$ by $\eta_{M_{i}}$; that is, $\eta_{M_{i}}=\eta_{M}\left(t_{i}\right), i=1, \ldots, m$. The problem $(7)$ is then approximated by the following uniformly discretized model:

$$
\eta_{M i}=\sum_{j=1}^{n} w_{j} H_{i j} a_{j}+\epsilon_{i}, \quad j=1, \ldots, n,
$$

where $w_{j}$ are weights for a quadrature rule, $a_{j}$ are a vector representation for the coefficient function $a(k)$, that is, $a_{j}=$ $a\left(k_{j}\right) ; \epsilon_{i}$ are an additive random noise, and the elements of $H_{i j}$ are given by

$$
H_{i j}= \begin{cases}\exp \left\{i\left(k_{j} X \pm \omega t_{i}\right)\right\}, & \text { for 2D problem, } \\ k_{j} J_{0}\left(k_{j} R\right) \cos \left(\omega t_{i}\right), & \text { for 3D problem. }\end{cases}
$$

Once the solution $a_{j}$ is obtained by inverting (9), the initial wave field can be reconstructed by the second step:

$$
\widehat{\eta}_{0}= \begin{cases}2 \mathscr{F}^{-1}(a), & \text { for 2D problem, } \\ \mathscr{H}^{-1}(a), & \text { for 3D problem, }\end{cases}
$$

where $\mathscr{F}^{-1}, \mathscr{H}^{-1}$ are the inverse Fourier transform and the inverse Hankel transform, respectively. The accent hat symbol means estimated value since it cannot be identical with the true value.
3.2. Instability. It is worth noting that the numerical system (9) is given by discretizing the integral operator (8), which is classified as Fredholm integral operator of the first kind. It is known that the approximation of such integral operator with a regular kernel yields a highly ill-conditioned system [7-11, 16-18]. As a result, the system (9) is ill conditioned and the inverse is greatly affected by small changes in input data (measurement). More specifically, this kind of system causes the following main difficulties in computational procedures [19]: (i) very large condition number of the matrix system, (ii) no guarantee about a useful solution from the replacement into a well-conditioned matrix.

Therefore, standard methods for obtaining the inverse solution cannot be simply used. Instead, more sophisticated methods should be applied to obtain a physically meaningful solution. In this study, we will address the difficulty by formulating the problem as an inference problem in an appropriately constructed stochastic model.

\section{Stochastic Model}

4.1. Bayesian Formulation. The aim of stochastic modeling is to derive the probability distribution, which is called the posterior probability density function (PPDF), for the unknown parameter conditional on available data according to Bayes' formula [18]:

$$
P\left(a \mid \eta_{M}\right) \propto P\left(\eta_{M} \mid a\right) P(a),
$$

where $P\left(\eta_{M} \mid a\right)$ is the likelihood function and $P(a)$ is the prior density function.

Bayesian formulation solves the ill-posed problem in a systematic way by modeling all parameters of interests as random variables with joint probability distributions. The probability distribution (12) can provide the information on the unknown parameter with the degree of confidence given the measurement.

The PPDF (12) can be specified through the appropriate probabilistic modeling for each component. Since the distribution (12) is proportional to the product of its likelihood function $P\left(\eta_{M} \mid a\right)$ and the prior density $P(a)$, the probabilistic modeling can be achieved by separately modeling these two probability distributions.

If we assume that the random error $\epsilon$ in the measurement is an additive Gaussian random noise with zero mean and standard deviation $\sigma$, the likelihood function $P\left(\eta_{M} \mid a\right)$ can be modeled $[12-15,18]$ as

$$
P\left(\eta_{M} \mid a\right) \propto\left(\sigma^{2}\right)^{-m / 2} \exp \left(-\frac{\left\|H a-\eta_{M}\right\|_{2}^{2}}{2 \sigma^{2}}\right),
$$

where $\|\cdot\|_{2}$ refers to Euclidean norm and $m$ is the number of measurements. It is worth emphasizing that other model can also be used to represent the likelihood function. But the Gaussian model (12) is the most commonly used since it is easy to handle and well fit the actual distribution.

Next, we need to consider the prior distribution $p(a)$ reflecting the knowledge of the unknowns before the data is measured. For the present study, there is no information 
about prior probability. For this case, a noninformative prior such as uniform distribution can be a possible choice. However, a noninformative prior may not provide sufficient regularity for the solution of an inverse problem which is severely ill posed. In this study, the following pairwise Markov random field (MRF) model, which has been successfully used in various applications [12-15], is used for the smoothness of the unknown coefficient function $a$ :

$$
p(a) \propto \lambda^{n / 2} \exp \left(-\frac{\lambda}{2} a^{T} W a\right)
$$

where the matrix $W \in \mathbb{R}^{n \times n}$ is determined by

$$
W_{i j}= \begin{cases}n_{i}, & i=j, \\ -1, & i \sim j, \\ 0, & \text { otherwise }\end{cases}
$$

where $n_{i}$ is the number of neighbors for the point $i$ and $i \sim j$ means that $i$ and $j$ are adjacent. The MRF is a model for a basis of contextual constraints in the prior distribution. It provides a way of synthesizing and capturing the characteristics of a stochastic process.

With the likelihood (13) and the prior models (14), the PPDF in (12) now can be expressed by

$$
\begin{aligned}
P\left(a \mid \eta_{M}\right) \propto & \left(\sigma^{2}\right)^{-m / 2} \exp \left(-\frac{\left\|H a-\eta_{M}\right\|_{2}^{2}}{2 \sigma^{2}}\right) \lambda^{n / 2} \\
& \times \exp \left(-\frac{\lambda}{2} a^{T} W a\right) .
\end{aligned}
$$

Here, it is worth noting that the expression (16) is dependent on some parameters $\sigma$ and $\lambda$, which are used for modeling likelihood function and the prior density. If these parameters are given, then the desired unknown parameter can be estimated by exploring the PPDF (16). However, in most cases, the choice of these parameters is also nondeterministic. In the stochastic model, these parameters are also modeled as random variables and have their own priors. The PPDF is then formulated as the following hierarchical model:

$$
\begin{aligned}
p\left(a, \lambda, \sigma \mid \eta_{M}\right) \propto & \left(\sigma^{2}\right)^{-\left(m / 2+\alpha_{2}+1\right)} \\
& \times \exp \left(-\frac{\left\|H a-\eta_{M}\right\|_{2}^{2}+2 \beta_{2}}{2 \sigma^{2}}\right) \lambda^{\left(n / 2+\alpha_{1}-1\right)} \\
& \times \exp \left(-\frac{\lambda\left(a^{T} W a+\beta_{1}\right)}{2}\right)
\end{aligned}
$$

where $\left(\alpha_{1}, \beta_{1}\right)$ is a pair of gamma distribution for $\lambda$ and $\left(\alpha_{2}, \beta_{2}\right)$ is a pair of inverse gamma distribution for the prior of $\sigma$.

4.2. Markov Chain Monte Carlo. Now the objective is to extract information on the unknown parameter $a$ from the constructed PPDF (17). The resultant posterior distribution can be analyzed using a Markov chain Monte Carlo (MCMC) sampling techniques. The PPDF (17) can be explored by the following hybrid algorithm which is designed by mixing Metropolis-Hastings steps in the Gibbs sampler [12-15].

(i) Initialize $a^{(0)}=\left\{a_{1}^{(0)}, a_{2}^{(0)}, \ldots, a_{n}^{(0)}\right\}, \lambda^{(0)}$, and $\sigma^{(0)}$,

(ii) For $i=0: N_{\mathrm{MCMC}}-1$

$$
\begin{aligned}
& \text { Sample } a_{1}^{(i+1)} \sim p\left(a_{1} \mid a_{2}^{(i)}, a_{3}^{(i)}, \ldots, a_{n}^{(i)}, \lambda^{(i)}, \sigma^{(i)}\right) \\
& \text { Sample } a_{2}^{(i+1)} \sim p\left(a_{2} \mid a_{1}^{(i+1)}, a_{3}^{(i)}, \ldots, a_{n}^{(i)}, \lambda^{(i)}, \sigma^{(i)}\right)
\end{aligned}
$$
:

$$
\begin{aligned}
& \text { Sample } a_{n}^{(i+1)} \sim p\left(a_{n} \mid a_{1}^{(i+1)}, a_{2}^{(i+1)}, \ldots, a_{n-1}^{(i+1)}, \lambda^{(i)}, \sigma^{(i)}\right) \\
& \text { Sample } u_{1} \sim U(0,1) \\
& \text { Sample } \lambda^{(*)} \sim q\left(\lambda^{(*)} \mid \lambda^{(i)}\right) \\
& \text { if } u_{1}<\min \left\{1, p\left(\lambda^{(*)} \mid a^{(i+1)}, \sigma^{(i)}\right) q\left(\lambda^{(i)} \mid \lambda^{(*)}\right) / p\left(\lambda^{(i)} \mid\right.\right. \\
& \left.\left.a^{(i+1)}, \sigma^{(i)}\right) q\left(\lambda^{(*)} \mid \lambda^{(i)}\right)\right\}, \lambda^{(i+1)}=\lambda^{(*)} \\
& \text { else } \lambda^{(i+1)}=\lambda^{(i)} \\
& \text { Sample } u_{2} \sim U(0,1) \\
& \text { Sample } \sigma^{(*)} \sim q\left(\sigma^{(*)} \mid \sigma^{(i)}\right) \\
& \text { if } u_{2}<\min \left\{1, p\left(\sigma^{(*)} \mid a^{(i+1)}, \lambda^{(i+1)}\right) q\left(\sigma^{(i)} \mid \sigma^{(*)}\right) / p\left(\sigma^{(i)} \mid\right.\right. \\
& \left.\left.a^{(i+1)}, \lambda^{(i+1)}\right) q\left(\sigma^{(*)} \mid \sigma^{(i)}\right)\right\}, \sigma^{(i+1)}=\sigma^{(*)} \\
& \text { else } \sigma^{(i+1)}=\sigma^{(i)}
\end{aligned}
$$

where the full conditionals are derived as

$$
\begin{gathered}
p(\lambda \mid a, \sigma) \sim G\left(\frac{n}{2}+\alpha_{1}, \frac{a^{T} W a+2 \beta_{1}}{2}\right), \\
p(\sigma \mid a, \lambda) \sim I G\left(\frac{m}{2}+\alpha_{2}, \frac{\left\|H a-\eta_{M}\right\|^{2}+2 \beta_{2}}{2}\right),
\end{gathered}
$$

where $G$ represents gamma distribution and $I G$ represents inverse gamma distribution.

\section{Numerical Experiments}

5.1. Example I: 2D Problem with Smooth-Type a $(k)$. As the first example, we consider the $2 \mathrm{D}$ problem of reconstructing the initial wave field with a smooth-type coefficient function $a(k)$. To illustrate the method, the following particular form of the initial surface disturbance is chosen:

$$
\eta_{0}=0.2 \sqrt{\pi} e^{-x^{2} / 4}
$$

The transform (4) of (19) yields the smooth-type coefficient function

$$
a(k)=0.1 e^{-x^{2}}
$$

Figure 2 shows the true initial disturbance (19) and the exact coefficient function (20).

The forward problem is first solved with the given initial wave field. The resulting nonsteady-state waves can be simply 


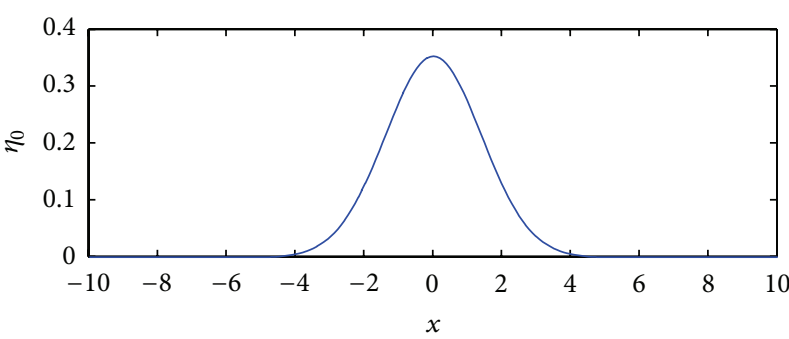

(a)

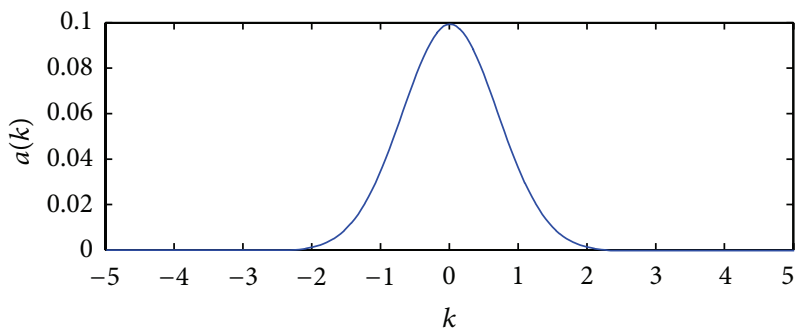

(b)

Figure 2: (a) True initial surface disturbance and (b) exact coefficient function in 2D example.

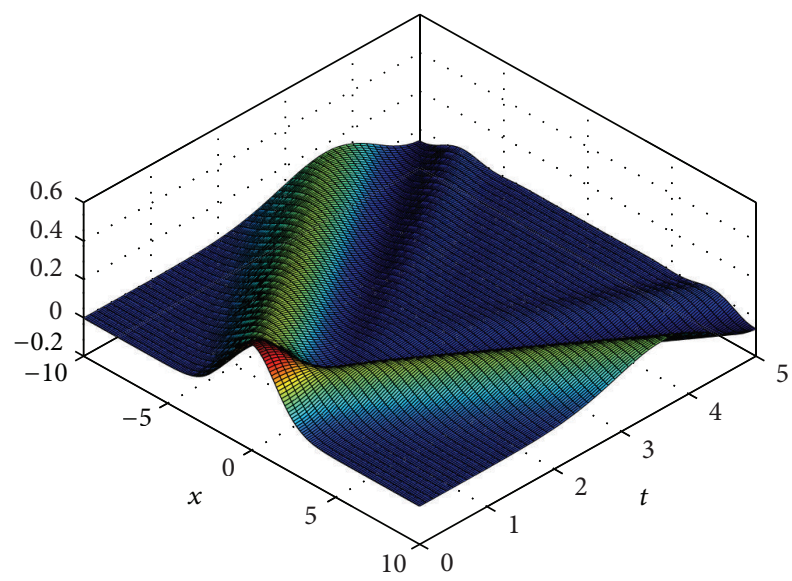

FIGURE 3: Spatial-time evolution of a 2D wave propagation.

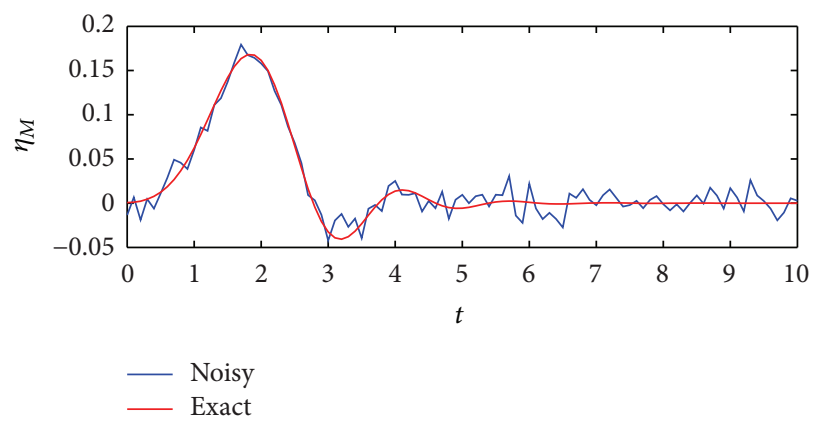

FIGURE 4: Time history of wave elevation collected at $x=5$.

computed through (5) with the coefficient function (20). Figure 3 shows the computed spatial-time evolution of a nonsteady-state water wave propagation generated by the initial wave disturbance (19). For the forward calculation, the integral operator is directly discretized by Simpson's integration rule. The water depth $h$ is normalized and $\Delta x=$ $0.1, \Delta t=0.1$ are considered as time and space steps on the physical domain $[-10,10] \times[0,10]$. To discretize the integral operator (8), the integration limit $N$ and $\Delta k$ are taken to be 5 and 0.05 , respectively.

From now, we consider the inverse problem of reconstructing the initial surface disturbance $\eta_{0}$ from the wave history data at a specific spatial point $x=X$. For illustrating purpose, a particular time history of wave elevation, $\eta_{M}$, is



FIGURE 5: The coefficient function obtained through the least squares estimate.

collected at $x=5$ and is shown in Figure 4. To mimic a practical situation, the Gaussian random noise $\epsilon$ is generated with the density $\sigma^{2}=0.01$ and added to the collected data. The resulting data with a random noise is considered as input data for the inverse problem.

As explained earlier, the system is severely ill posed and thus the inverse is mathematically and numerically much more challenging than the forward calculation. To examine the cause and the degree of instability of the system, the discretized system is decomposed as

$$
\eta_{M}=\sum_{i=1}^{n} \sigma_{i}\left(v_{i}^{T} a\right) u_{i}
$$

where $\sigma_{i}$ 's are the singular values ordered, $\sigma_{1} \geq \sigma_{2} \geq \cdots>$ $\sigma_{\infty}$, and $\left\{u_{i}\right\}_{1}^{n},\left\{v_{i}\right\}_{1}^{n}$ are orthonormal singular vectors. The preceding decomposition is well known as the singular value decomposition [16-19]. The unknown $a$ can then be obtained by the least squares estimate, that is, $\min _{a}\left\|K a-\eta_{M}\right\|_{2}$ :

$$
a_{\mathrm{LSQ}}=\sum_{i=1}^{n} \frac{u_{i}^{T} \eta_{M}}{\sigma_{i}} v_{i} .
$$

With the measurement data in Figure 4, we first obtained the inverse solution using the least squares estimate (22). It can be seen from Figure 5 that the computed inverse solutions are totally unstable and thus useless. The cause and the degree of instability in the inverse solution can be understood by analyzing the behavior of singular systems, more specifically, 


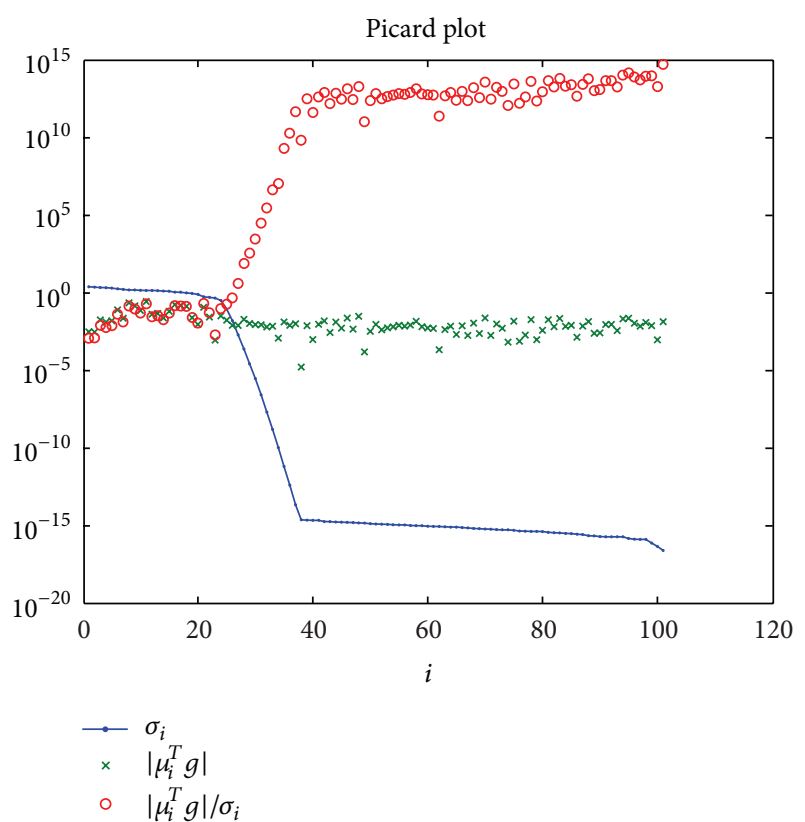

Figure 6: Picard plots.



Figure 7: Posterior mean estimate for the coefficient function.

each component in the right-hand-side of (22). Figure 6 shows the Picard plot [19] that shows behaviors of $\left|u_{i}^{T} \eta_{M}\right|, \sigma_{i}$, and the ratio $\left|u_{i}^{T} \eta_{M}\right| / \sigma_{i}$. It can be found that the coefficients $\left|u_{i}^{T} \eta_{M}\right|$ become larger than most of singular values $\sigma_{i}$. Thus, the least squares solution for $a$, which is the sum of the ratio $\left|u_{i}^{T} \eta_{M}\right| / \sigma_{i}$, is dominated by the small singular values. This explains the reason for the unstable solution in Figure 5. The instability, frequent sign changes, may be accelerated and amplified with the increasing amount of noise in data.

With this analysis in mind, we next construct the stochastic inverse model (17) in terms of the measured wave history data. To extract useful information from the probability density, MCMC algorithm described in Section 4 is used. The initial guess for $\lambda, \sigma$, and $a$ are taken to be $1,0.1$, and zero vector, respectively. The pairs of parameters $\left(\alpha_{1}, \beta_{1}\right)$, $\left(\alpha_{2}, \beta_{2}\right)$ are taken to be $\left(10^{-1}, 10\right),\left(10^{-1}, 10\right)$, respectively. The total number of samples $N_{\mathrm{MCMC}}$ for the algorithm was taken to be 50,000 and the last 25,000 samples were used to estimate statistics for $a$ since it allows the beginning of

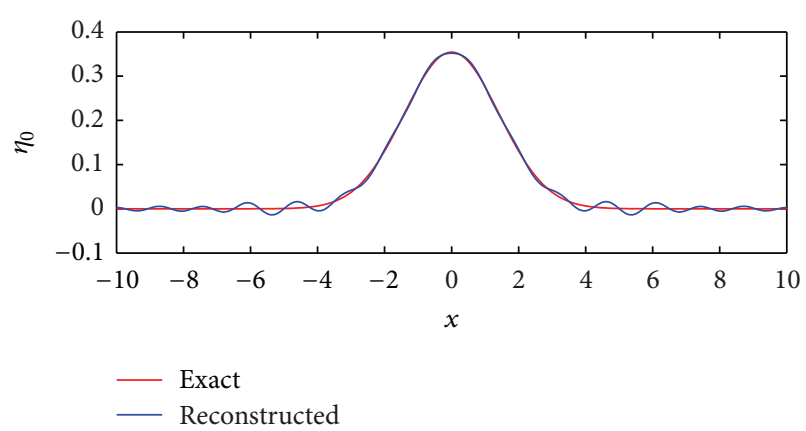

FIGURE 8: Reconstructed initial wave disturbance $\eta_{0}$ for the $2 \mathrm{D}$ example.

the Markov chain. After obtaining random samples from the MCMC algorithm, it is necessary to examine the issues such as convergence and mixing of the chain to determine whether simulated set of samples can reasonably be treated as a set of random realizations from the target distribution. In this study, marginal trace plots are used to check if the chain works properly. All the components have the stationary distribution as its marginal distribution.

The true and estimated coefficient functions are shown in Figure 7. The upper and lower dotted lines denote the 95\% credible interval. The credible interval quantifies the degree of uncertainties in the solution. The mean estimate of the posterior density is fairly stable and accurate compared with the results in Figure 8. Finally, the initial wave field $\eta_{0}$ is reconstructed using the posterior mean of the coefficient function. The reconstructed initial surface disturbance in Figure 8 represents accurate approximation to the true initial disturbance.

5.2. Example II: 3D Problem with Nonsmooth-Type a(k). As the second example, we consider the $3 \mathrm{D}$ problem of reconstructing the initial wave field with a nonsmooth-type coefficient function $a(k)$, which is believed to be a more challenging case than the smooth type. For the purpose, we first specify the initial surface disturbance for $3 \mathrm{D}$ problem as

$$
\eta_{0}=\frac{1}{r} J_{1}(r)
$$

where $J_{1}$ is Bessel function of the first kind of order one and $r=\sqrt{x^{2}+y^{2}}$. The transform of (21) yields the nonsmooth type coefficient function:

$$
a(k)= \begin{cases}1, & 0 \leq k \leq 1 \\ 0, & 1<k<\infty\end{cases}
$$

Figure 9 shows the true initial disturbance (21) and the exact coefficient function (22).

In the similar manner of the previous example, the forward problem is first solved with the given initial surface disturbance. For the calculation, the water depth $h$ is normalized and $\Delta r=0.1, \Delta t=0.1$ are considered as time and space steps on the physical domain $[0,10] \times[0,10]$. When discretizing the integral operator (8), the integration limit $N$ 


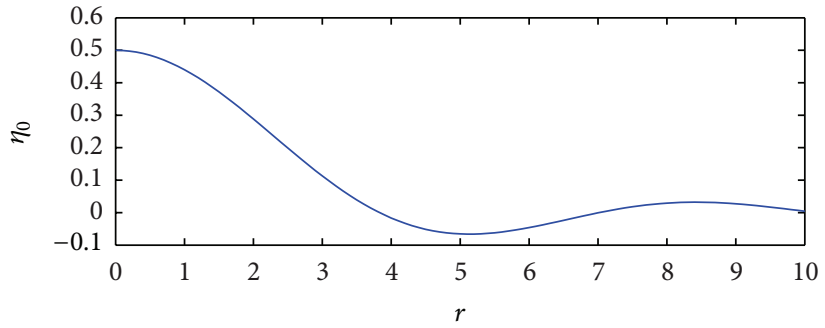

(a)



(b)

Figure 9: (a) True initial wave disturbance and (b) exact coefficient function in 3D example.

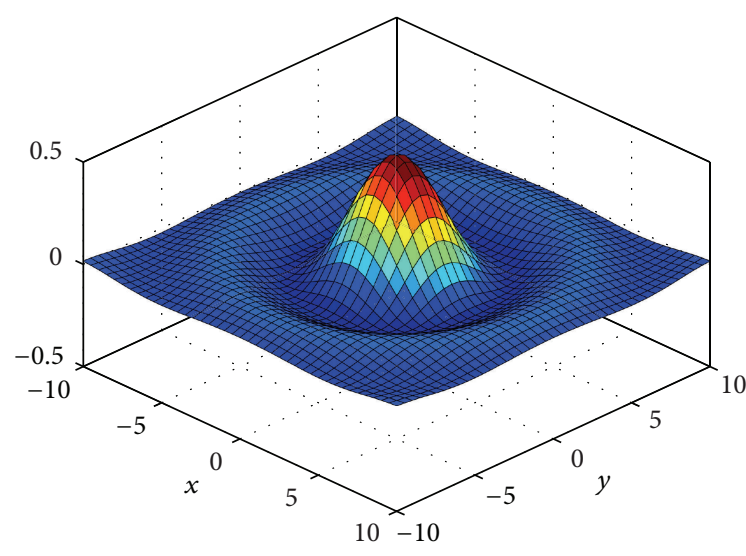

(a) $t=0$

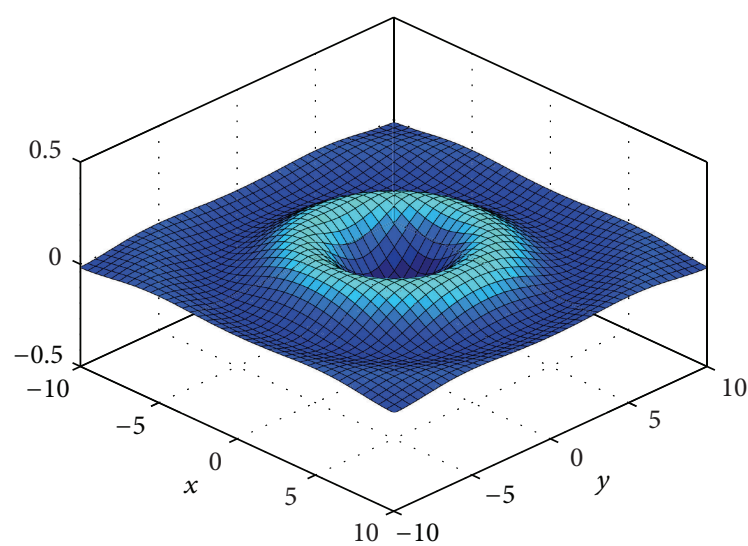

(c) $t=1$

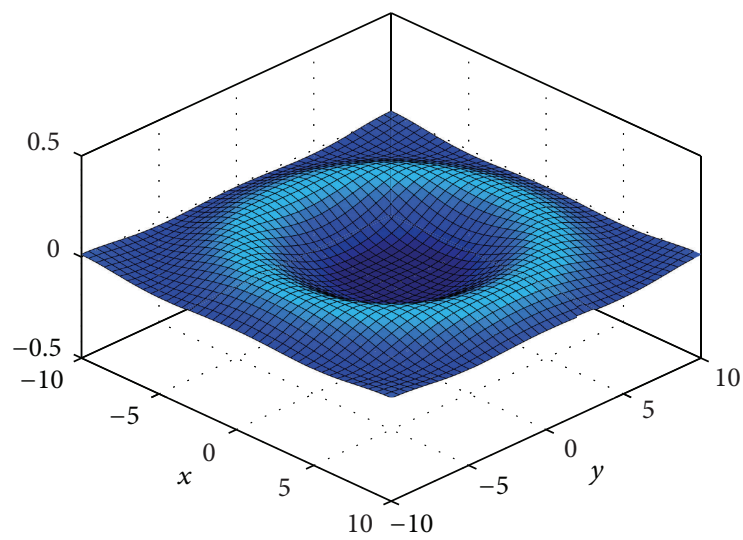

(e) $t=2$

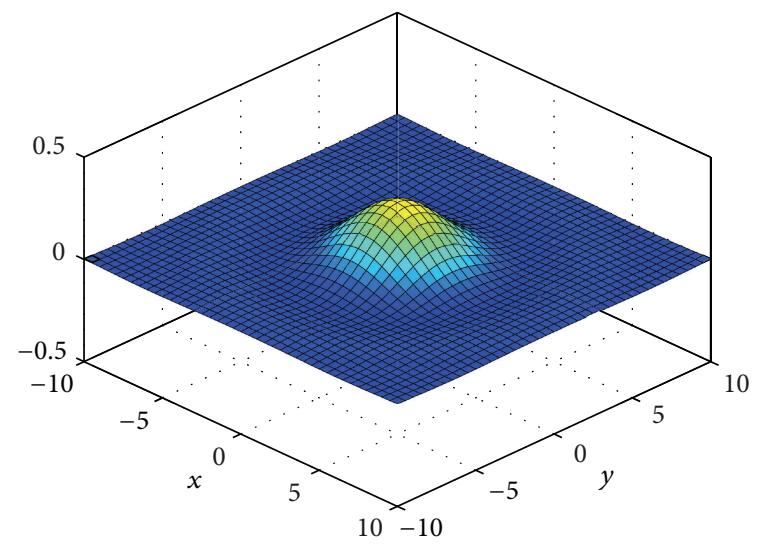

(b) $t=0.5$

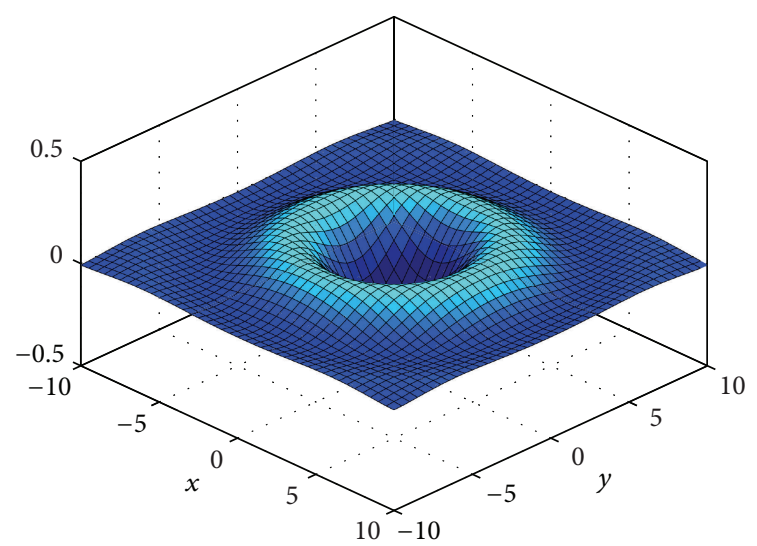

(d) $t=1.5$



(f) $t=2.5$

FIGURE 10: Spatial-time evolution of 3D wave propagation. 


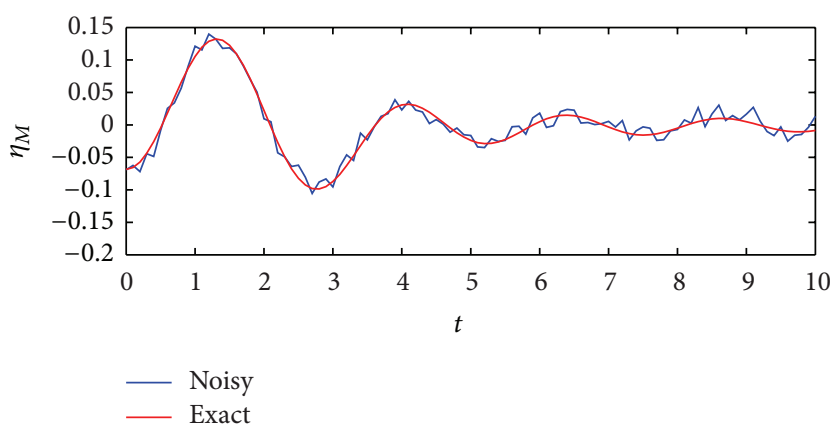

FIGURE 11: Time history of wave elevation collected at $r=5$.

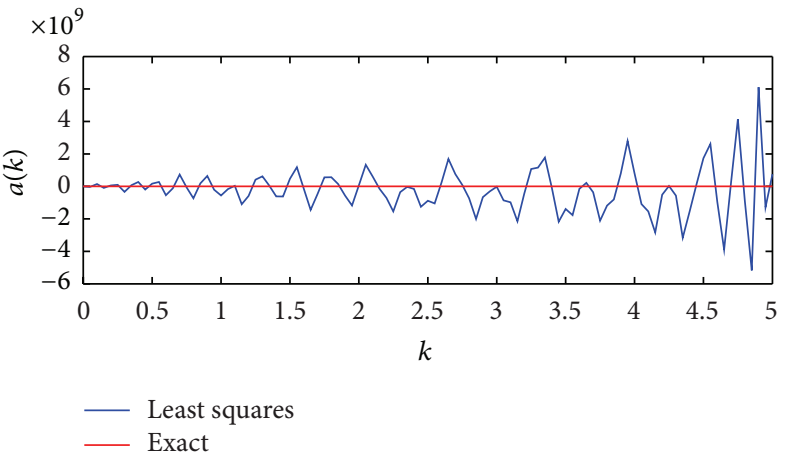

(a)

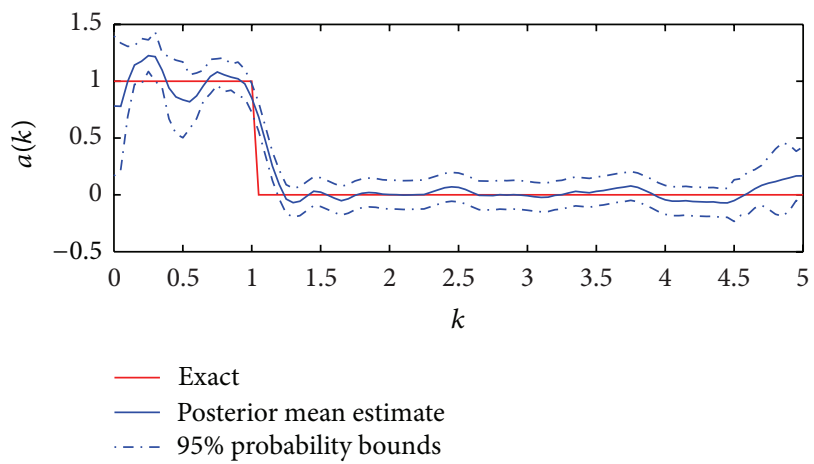

(b)

FIGURE 12: (a) Least squares estimate and (b) Posterior mean estimate of the MCMC samples for the coefficient function.

and $\Delta k$ are taken to be 5 and 0.05 , respectively. Figure 10 shows the computed spatial-time evolution for 3D problem.

The wave history data in Figure 11, which is recorded at $r=5$, is used to illustrate the reconstructing procedure. To generate noisy data, the random noise $\epsilon$ with the variance $\sigma^{2}=0.01$ is added to the collected data. With this measurement, the coefficient function is estimated through the MCMC algorithm. The same conditions as in the first example are used for the MCMC algorithm. The posterior mean estimate of the simulated random samples is shown in Figure 12. For the purpose of comparison, the least squares estimation of the coefficient function is also illustrated. The result indicates that the posterior mean is in good agreement with the exact coefficient function while the result from least squares estimate is totally unstable. Using this posterior mean estimate, the initial wave field is reconstructed and shown in Figure 13. It can be found that the estimated result accurately approximate to the true initial disturbance.

It is also worth to compare the performances of the existing algorithms and the stochastic inverse approach illustrated in this paper to underline the advantages and disadvantages. For this purpose, Tikhonov regularization method $[8,11,16-$ 19], which is known as a representative deterministic inverse approach, was employed to solve the second example with the same conditions. Tikhonov regularized solution for $a(k)$ is the minimizer of the functional

$$
F(a)=\left\|H a-\eta_{M}\right\|_{2}^{2}+\delta\|a\|_{2}^{2}
$$

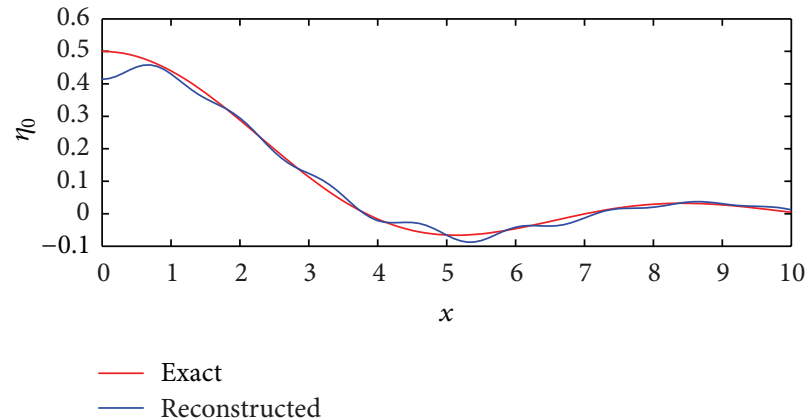

FIGURE 13: Reconstruction of the initial wave disturbance for the 3D example.

where $\delta>0$ is the regularization parameter.

In deterministic inverse approach, the choice of the regularization parameter is very important because it controls the performance of the regularization. However, selecting an appropriate regularization parameter is not trivial since it depends on both the regularization method itself and the mathematical model of interest in a very complex way. Generally, the regularization parameter is selected by the heuristic method. First, the inverse problem is solved with a set of regularization parameters. The solution remains practically unchanged for a certain range of the parameter. The regularization parameter can then be chosen from this range. Figure 14 shows the Tikhonov regularized solutions to 




(a) $\delta=10^{-1}$

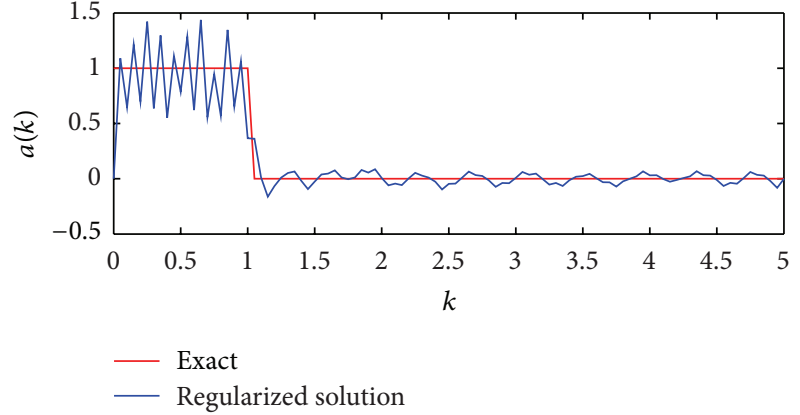

(b) $\delta=10^{-2}$

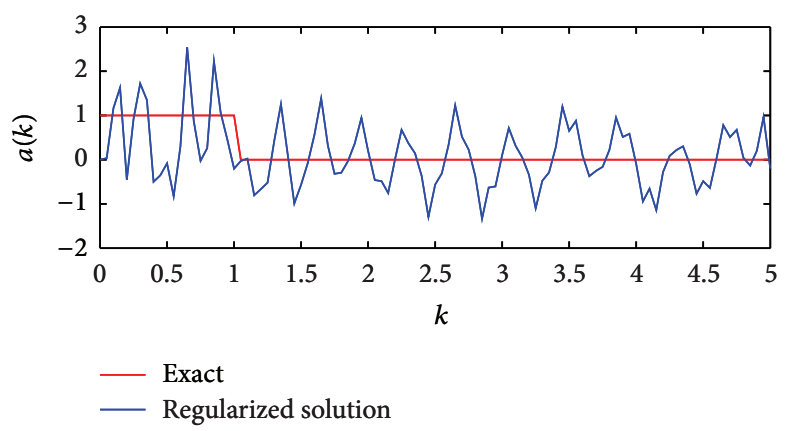

(c) $\delta=10^{-3}$

FIGURE 14: Tikhonov regularized solution for three different regularization parameters.

three different regularization parameters. It can be observed that the poor solution is obtained when the regularized solution to the regularization parameter is large. Furthermore, the solution loses its stability when the regularization parameter is small. Therefore, it is necessary to pay attention to the behavior of the regularized solution, when choosing the regularization parameter. It is seen by comparing with Figure 12 that the proposed stochastic inverse approach is smoother than the Tikhonov method.

Compared with the deterministic inverse methods for inverse problems, the proposed stochastic inverse approach has some advantages. Firstly, it provides a natural framework for quantifying the uncertainties by considering a complete probabilistic description of the inverse problem of interest. Generally, there exist many possible candidates for the solution, because of the ill-posed nature of the inverse problem. But, the deterministic inverse methods provide only a single estimate taken from these possible candidates based on the choice of the regularization parameter. Secondly, it also provides a flexible way to choose a nondeterministic parameter by formulating the hierarchical model.

However, there also exist disadvantages. In this work, a computational framework for reconstructing the initial wave field was built based on the Markov chain Monte Carlo technique. In computational mathematics, the efficient computation is important. The key behind the Markov chain is to generate samples from the target distribution based on the iteration. To ensure the chain convergence, quite large numbers of samples are required. Thus, it is expected that the solver will be computationally very expensive because the computational algorithm of the stochastic inverse model requires the direct simulation. In contrast, the deterministic inverse methods can remarkably reduce the computational time by avoiding the time-consuming iterations.

\section{Conclusions}

The conclusions can be summarized as follows.

(1) The fluid motion has been taken to be homogeneous, incompressible, and inviscid such that the velocity potential exists and satisfies Laplace's equation. On this basis, it has been shown that, for a given initial surface disturbance, the surface wave motion can be mathematically expressed as an integral transform, which can be used to compute the time-spatial evolution of the wave motion.

(2) The inverse problem of reconstructing the initial surface disturbance was formulated with a time history data of surface elevation which is collected at a specific spatial point. The inverse problem requires solving an ill-posed system that causes numerical instability. It was shown that, for such system, a standard method such as least squares estimate fails to obtain the stable solution. To address the difficulty, the system was formulated as an inference problem in an appropriately constructed stochastic model.

(3) The reconstruction procedure based on the stochastic inverse model has been evaluated through an analysis of some digitally simulated data for numerical examples related to two- and three-dimensional fluid 
motion. It has been shown that the proposed procedure can be used to not only reconstruct the initial surface disturbance but also detect uncertainties in solution arising from measurement error.

In this study, the quality of the reconstruction has been illustrated by two examples, where the simulated data fulfilled the assumptions about the noise. Future work on this study will also include the application of real data for ensuring robustness of the method.

\section{References}

[1] C. C. Mei, The Applied Dynamics of Ocean Surface Waves, World Scientific, London, UK, 1989.

[2] J. J. Stoker, Water Waves, Wiley Classics Library, John Wiley \& Sons, New York, NY, USA, 1992.

[3] G. B. Whitham, Linear and Nonlinear Waves, Pure and Applied Mathematics, John Wiley \& Sons, New York, NY, USA, 1999.

[4] B. L. Mehaute and S. Wang, Waves Generated by Underwater Explosion, World Scientific, 1996.

[5] J. Lighthill, Waves in Fluids, Cambridge University Press, Cambridge, UK, 1978.

[6] A. R. Osborne, Nonlinear Ocean Waves and the Inverse Scattering Transform, vol. 97 of International Geophysics Series, Elsevier/Academic Press, Boston, Mass, USA, 2010.

[7] T. S. Jang, S. H. Kwon, and B. J. Kim, "Solution of an unstable axisymmetric Cauchy-Poisson problem of dispersive water waves for a spectrum with compact support," Ocean Engineering, vol. 34, no. 5-6, pp. 676-684, 2007.

[8] T. S. Jang and S. L. Han, "Application of Tikhonov's regularization to unstable water waves of the two-dimensional fluid flow: spectrum with compact support," Ships and Offshore Structures, vol. 3, no. 1, pp. 41-47, 2008.

[9] T. S. Jang, S. L. Han, and T. Kinoshita, "An inverse measurement of the sudden underwater movement of the sea-floor by using the time-history record of the water-wave elevation," Wave Motion, vol. 47, no. 3, pp. 146-155, 2010.

[10] T. S. Jang, H. G. Sung, and J. Park, "A determination of an abrupt motion of the sea bottom by using snapshot data of water waves," Mathematical Problems in Engineering, vol. 2012, Article ID 472575, 16 pages, 2012.

[11] S. H. Kwon, C. H. Kim, and T. S. Jang, "An identification of wave propagation based on a single-point measurement," Ocean Engineering, vol. 34, no. 10, pp. 1405-1412, 2007.

[12] J. Wang and N. Zabaras, "Hierarchical Bayesian models for inverse problems in heat conduction," Inverse Problems, vol. 21, no. 1, pp. 183-206, 2005.

[13] J. Wang and N. Zabaras, "A Markov random field model of contamination source identification in porous media flow," International Journal of Heat and Mass Transfer, vol. 49, no. 5-6, pp. 939-950, 2006.

[14] S. L. Han and T. Kinoshita, "Nonlinear damping identification in nonlinear dynamic system based on stochastic inverse approach," Mathematical Problems in Engineering, vol. 2012, Article ID 574291, 20 pages, 2012.

[15] S. L. Han and T. Kinoshita, "Investigation of a stochastic inverse method to estimate an external force: applications to a wavestructure interaction," Mathematical Problems in Engineering, vol. 2012, Article ID 175036, 25 pages, 2012.
[16] A. Kirsch, An Introduction to the Mathematical Theory of Inverse Problems, vol. 120 of Applied Mathematical Sciences, Springer, New York, NY, USA, 1996.

[17] C. W. Groetsch, Inverse Problems in the Mathematical Sciences, Informatica International, 1993.

[18] J. Kaipio and E. Somersalo, Statistical and Computational Inverse Problems, vol. 160 of Applied Mathematical Sciences, Springer, New York, NY, USA, 2005.

[19] P. C. Hansen, Rank-Deficient and Discrete ill-Posed Problems, SIAM Monographs on Mathematical Modeling and Computation, Society for Industrial and Applied Mathematics (SIAM), Philadelphia, Pa, USA, 1998. 




Advances in

Operations Research

mansans



The Scientific World Journal
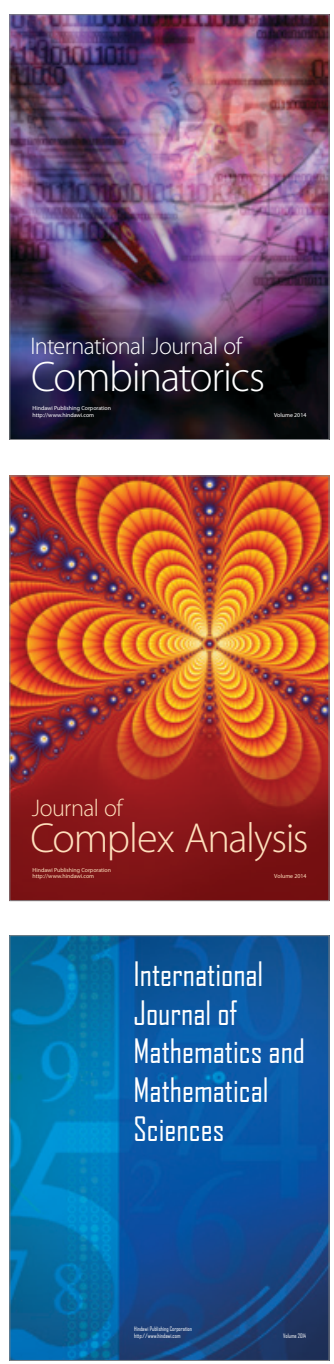
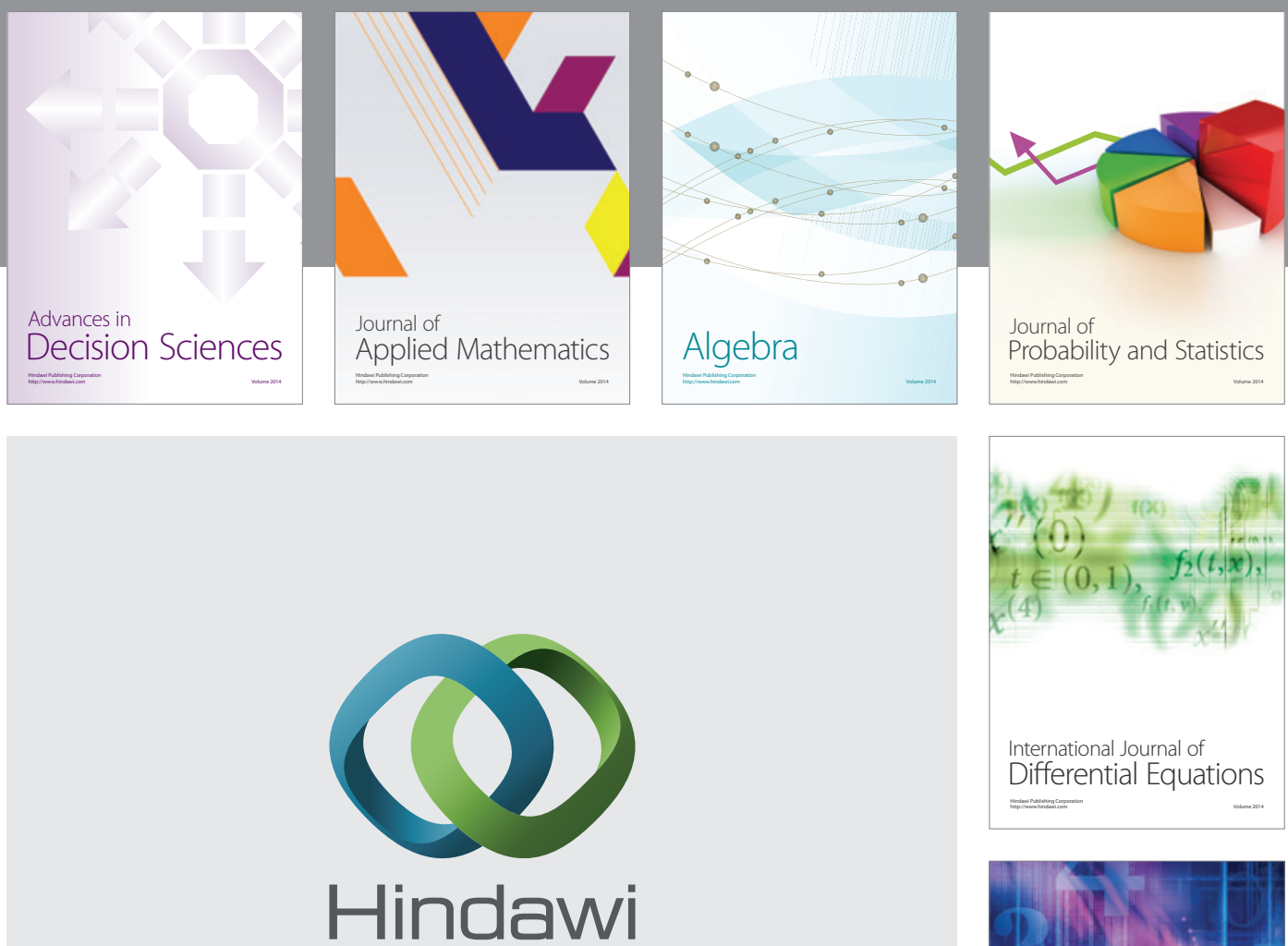

Submit your manuscripts at http://www.hindawi.com
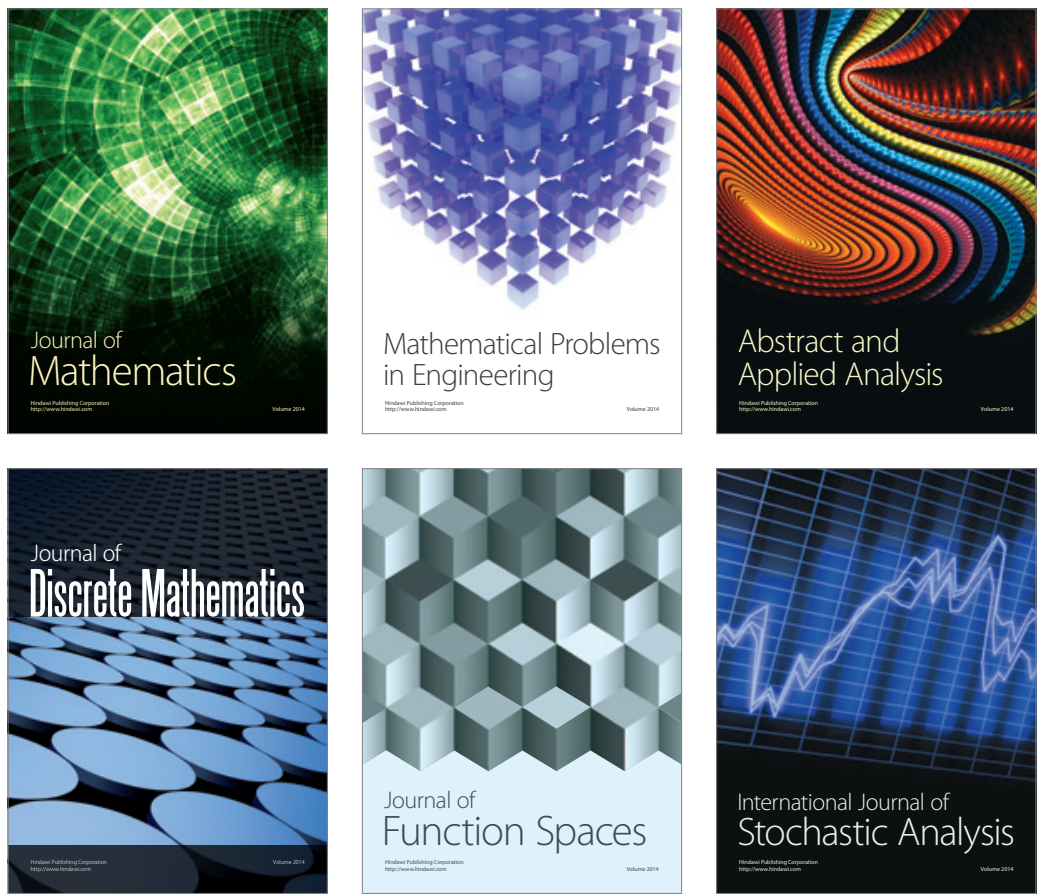

Journal of

Function Spaces

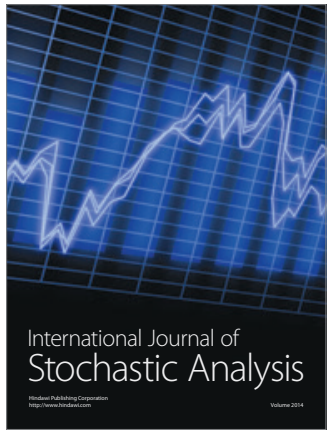

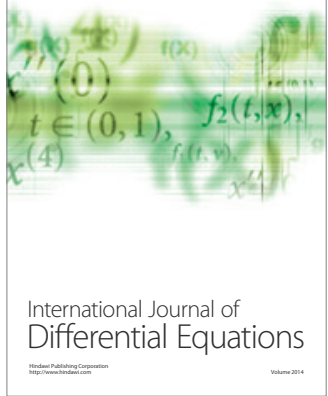
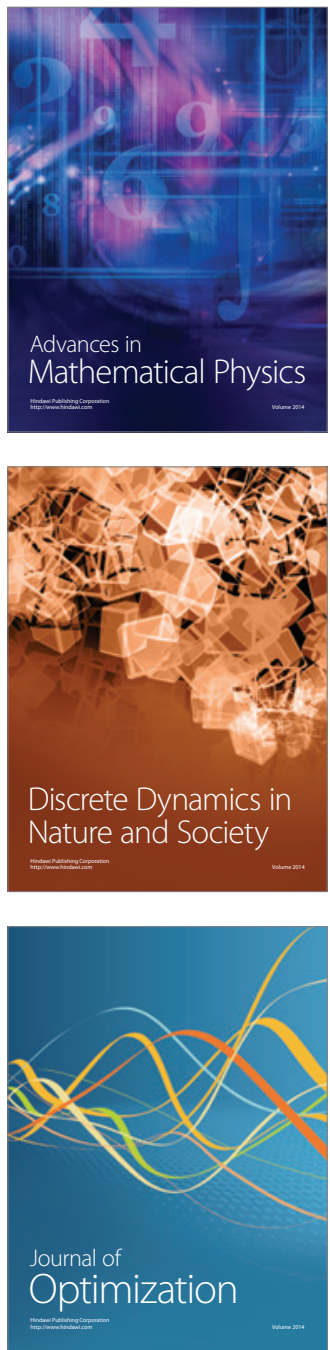